\title{
A Young Female with Fever and Proteinuria
}

\author{
Manish Rathi ${ }^{1}$, Joyita Bharati ${ }^{2},{\text { Adarsh } \mathrm{MB}^{3} \text {, Ritambhra Nada }}^{4}$, Kusum Sharma ${ }^{5}$, Aman Sharma ${ }^{6}$, Krishan L Gupta ${ }^{7}$
}

\begin{abstract} both simultaneously. resolution of disseminated tuberculosis.

Keywords: Case report, Granuloma, Lupus nephritis, Tuberculosis.

\section{BACKGROUND}

Lupus patients are immunocompromised either due to disease activity or due to immunosuppressive treatment. Lupus nephritis is an additional risk factor for TB as these patients are usually treated with higher doses of immunosuppression. This is the first report describing concomitant proliferative lupus nephritis with tubercular granulomatous tubulointerstitial nephritis (GIN) in a lupus patient with disseminated TB and the challenges faced during the diagnosis and management.
\end{abstract}

Aim: We aim to describe an unusual association between systemic lupus erythematosus (SLE) and tuberculosis and the challenges of managing

Background: Systemic lupus erythematosus is an autoimmune disease with an underlying defect in innate immunity and a predisposition for various infections such as tuberculosis. On the contrary, tuberculosis is known to trigger the onset and/or flare of SLE.

Case description: We report a young female with florid manifestations of SLE with proliferative lupus nephritis and coexisting disseminated tuberculosis complicated by tubercular granulomatous tubulointerstitial nephritis (GIN). She was treated with oral prednisolone (1 mg/kg/ day) and mycophenolate mofetil (MMF) with antituberculous drugs. Following 6 months of therapy, she achieved complete remission and

Conclusion: Molecular methods help in appropriate diagnosis of renal tuberculous granulomas. This report discusses the interactions between tuberculosis and SLE and also reviews therapeutic options of immunosuppression in active lupus with concomitant tuberculosis.

Journal of Postgraduate Medicine Education and Research (2020): 10.5005/jp-journals-10028-1348

\section{Case Description}

A 29-year-old lady with no known comorbidities or renal disease presented with a history of intermittent fever, photosensitivity, and joint pain for one year. She also had easy fatigability and progressively increasing exertional shortness of breath for six months. She had decreased appetite and weight loss of $15 \mathrm{~kg}$ during the last one year. There was shortness of breath on exertion for 6 months, which gradually increased for one month. There was no history of cough or chest pain. She had two first-trimester abortions.

On examination, she had tachypnea (respiratory rate: 28/ minute) and tachycardia (pulse rate: 140/minute) with normal blood pressure at presentation. There was severe pallor, and generalized lymphadenopathy involving cervical, axillary, and epitrochlear nodes. Her body mass index was $19.2 \mathrm{~kg} / \mathrm{m}^{2}$. Investigations showed hemoglobin of $6.2 \mathrm{~g} / \mathrm{dL}$, normochromic and normocytic erythrocytes with a corrected reticulocyte count of $1.2 \%$ and negative hemolytic workup. Serum creatinine was $0.9 \mathrm{mg} / \mathrm{dL}$, and serum albumin was $1.86 \mathrm{~g} / \mathrm{dL}$ with a 24-hour urine protein excretion of $1.052 \mathrm{~g} /$ day. Urine dipstick was positive for albumin $(1+)$, and microscopy was suggestive of 6-8 erythrocytes/high-power field, but no casts. Her Mantoux test was negative. Antinuclear antibody was 3+ with diffuse pattern on indirect immunofluorescence with positive anti-dsDNA (>379 IU/mL), and low C3 $(21.6 \mathrm{mg} / \mathrm{dL})$ and C4 $(2.98 \mathrm{mg} / \mathrm{dL}$ ) values. A renal ultrasound revealed normal-sized kidneys and normal echogenicity. A renal biopsy was done for
1,2,7Department of Nephrology, Postgraduate Institute of Medical Education and Research, Chandigarh, India

${ }^{3,6}$ Department of Internal Medicine, Postgraduate Institute of Medical Education and Research, Chandigarh, India

${ }^{4}$ Department of Histopathology, Postgraduate Institute of Medical Education and Research, Chandigarh, India

${ }^{5}$ Department of Medical Microbiology, Postgraduate Institute of Medical Education and Research, Chandigarh, India

Corresponding Author: Manish Rathi, Department of Nephrology, Postgraduate Institute of Medical Education and Research, Chandigarh, India, Phone: +0172-2756734, e-mail: drmanishrathi2000@yahoo.co.in How to cite this article: Rathi M, Bharati J, MB Adarsh, et al. A Young Female with Fever and Proteinuria. J Postgrad Med Edu Res 2020;54(1):12-14.

Source of support: Nil

Conflict of interest: None

possibility of lupus nephritis. Light microscopy details are shown in Figure 1.

The kidney biopsy consisted of 7 glomeruli, 3 out of which were noted for mesangial expansion and endocapillary proliferation, with 2 having hyaline thrombi (Fig. 1). Multiple epithelioid cell granulomas with Langhan's giant cells and large areas of necrosis were seen in the interstitium causing destruction of tubules (Fig. 2). There were no eosinophils, or interstitial fibrosis noted. There was evidence of vasculitis in smaller arteries seen. The immunofluorescence analysis revealed intense $(3+)$ positivity for antisera specific for $\lg G$, $\lg A$, lgM, $\mathrm{C} 3, \mathrm{C} 1 \mathrm{q}, \mathrm{K}$, and $\lambda$ light chains in subendothelium and mesangium, denoting full-house staining pattern (Fig. 3). Extraglomerular deposits for IgG, IgA, kappa, and lambda were noted along the blood vessels. The diagnosis was confirmed to be lupus nephritisclass III (A) with evidence of vasculitis and necrotizing interstitial granulomas. Although acid-fast bacilli (AFB) stain was negative, 


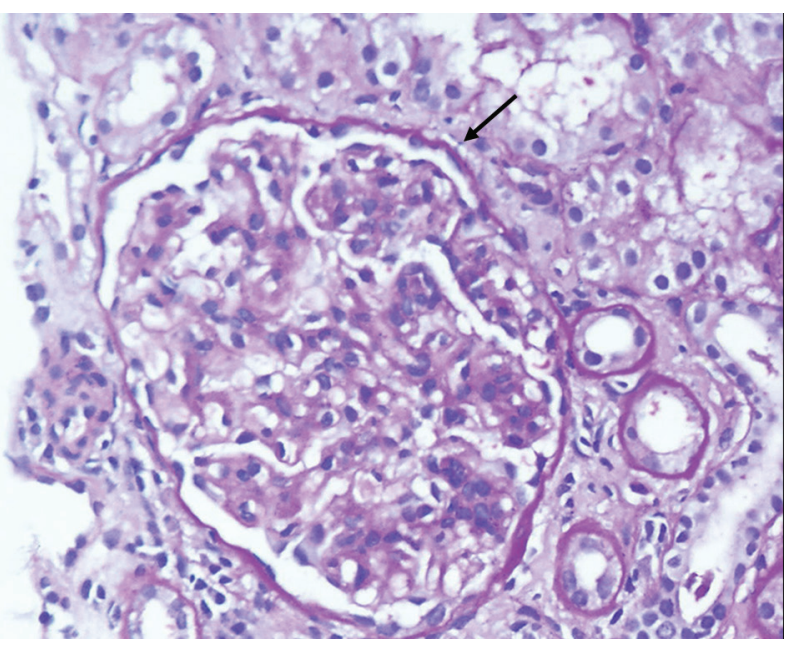

Fig. 1: Hematoxylin and eosin $\times 200 —$ glomerulus showing endocapillary proliferation (arrow)
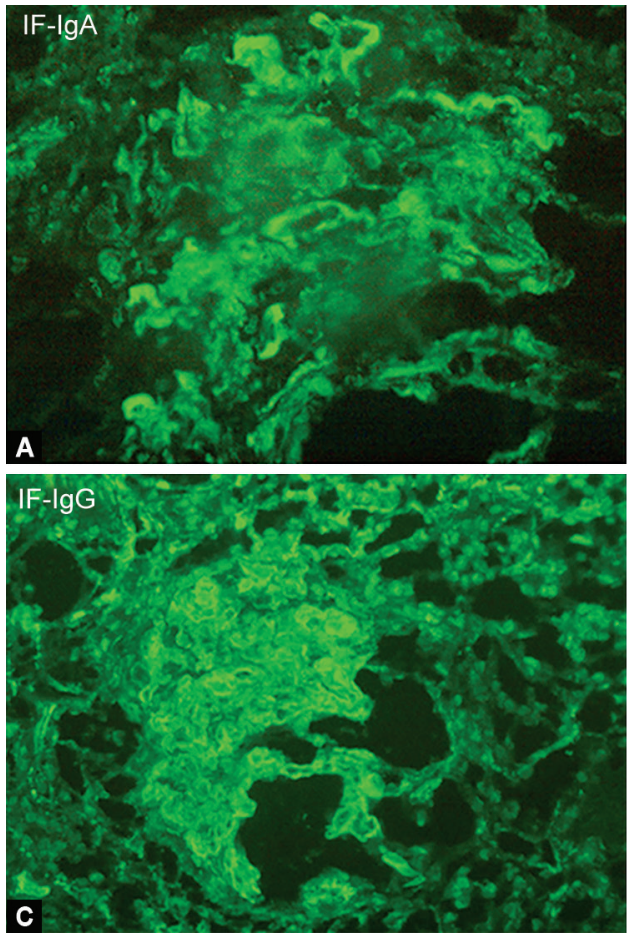

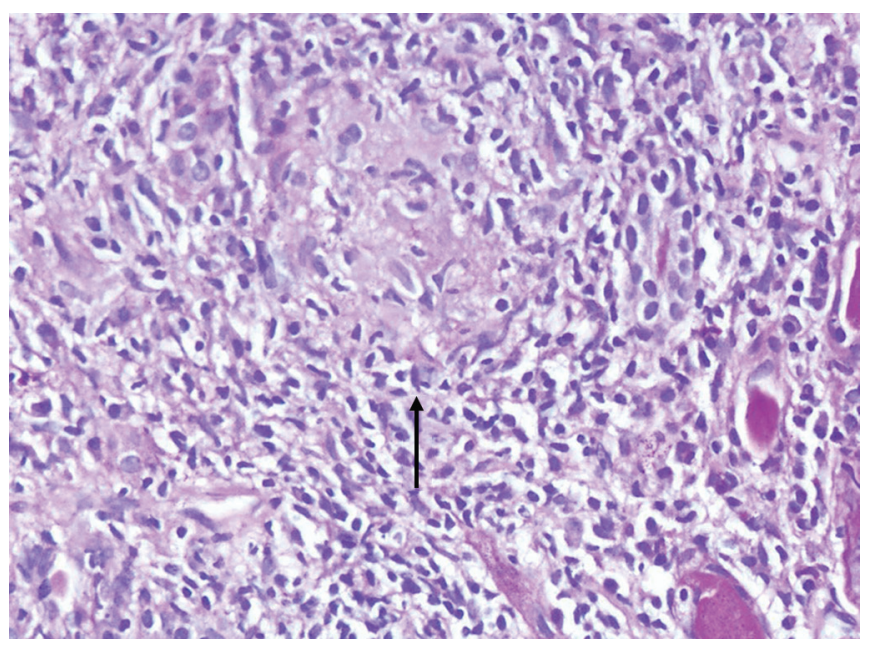

Fig. 2: Hematoxylin and eosin $\times 200$-epithelioid granuloma in the interstitium (arrow)
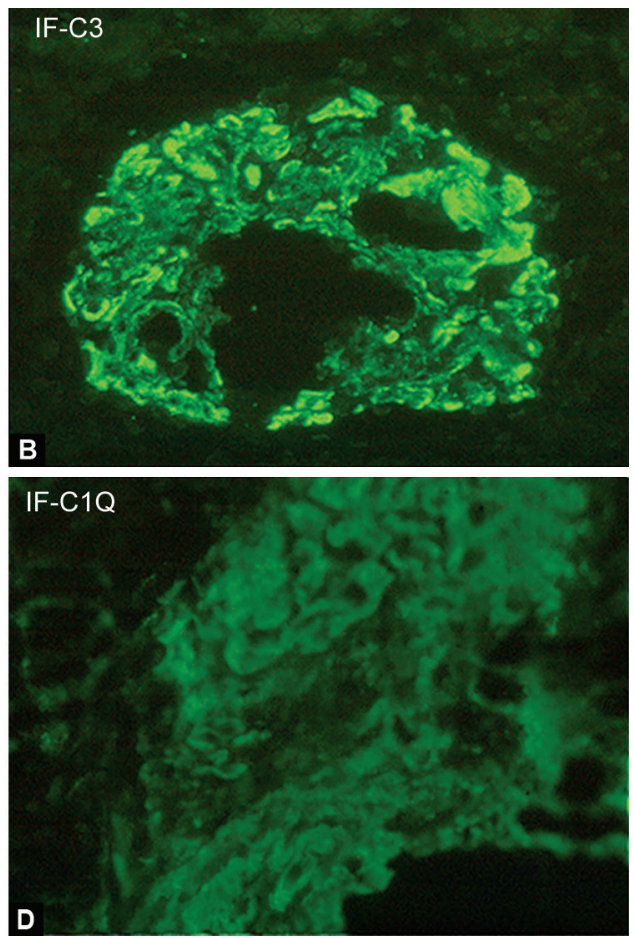

Figs 3A to D: Direct immunofluorescence-glomerular capillary wall and mesangial stain for $\lg G, \lg A, C 1 q$ and C3

mycobacterial protein was detected using multiplex polymerase chain reaction (PCR) in kidney tissue (Fig. 3: inset). There was evidence of disseminated tuberculosis in the form of bilateral lung consolidation with cavity formation, peritoneal enhancement with ascites, and retroperitoneal lymphadenopathy in the contrast enhanced computed tomography (CECT) of chest and abdomen. CT guided lung biopsy revealed interstitial epithelioid granulomas with multinucleated giant cells, and caseous necrosis and stain for AFB was positive, confirming the diagnosis of tuberculosis (Fig. 4). Patient was diagnosed with systemic lupus erythematosus (SLE) with renal and hematological involvement with disseminated tuberculosis and was started on corticosteroids ( $1 \mathrm{mg} / \mathrm{kg} /$ day) with antituberculous treatment. Mycophenolate mofetil (MMF) at the dose of $2 \mathrm{~g}$ per day was introduced after initial 1 month of antituberculous therapy for underlying lupus nephritis. After 6 months of follow-up, patient achieved complete remission in proteinuria with normal renal function.

\section{Discussion}

Systemic lupus erythematosus and TB infection are known to interact and mimic each other clinically, often leading to delayed diagnosis of active TB infection in such patients. The incidence and severity of TB are higher in lupus patients, specifically in endemic countries, which is attributable to the defective immunity in SLE patients and higher exposure to infection. ${ }^{1}$ Tuberculosis can, in turn, act as a trigger for SLE owing to shared antigens between 


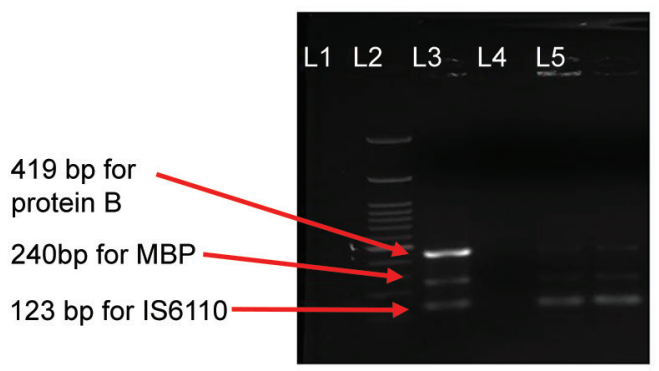

L1-100bp molecular marker, L2- DNA from positive control H37RV which is positive from all the three bands, L3- negative control, L4,5- DNA extracted from renal tissue positive for all bands of MTB

Fig. 4: Gel picture of multiplex PCR (kidney tissue): L1—100 bp molecular marker, L2 - positive control with 419 bp (protein b band), 240 bp (MBP) and 123 bp (IS6110 band), L3 - negative control, L4 and L5-positive patient sample

Mycobacterium tuberculosis and autoantigens in patients. ${ }^{2}$ Lupus patients with TB are also at increased risk of dissemination. ${ }^{2}$ Our patient presented with disseminated tuberculosis with first presentation of active SLE simultaneously. She was found to have proliferative lupus nephritis with interstitial necrotizing granulomas. Granulomatous tubulointerstitial nephritis is a rare entity comprising only $0.5-0.9 \%$ of all renal biopsies. ${ }^{3,4}$ Whereas the commonest cause worldwide is drugs, ${ }^{5}$ TB was reported as the most common etiology in the Indian subcontinent. ${ }^{4}$ Furthermore, SLE has also been described as a cause of GIN. ${ }^{4}$ Joss et al. ${ }^{3}$ found the poor role of histological features to discriminate against various causes of GIN. Moreover, definitive diagnosis of tuberculous GIN is often difficult with conventional methods such as urine culture or stain for AFB and caseous necrosis in the granulomas. Use of molecular methods such as multiplex PCR for mycobacterial protein in kidney tissue has been shown to yield positive results in tuberculous GIN earlier. ${ }^{6}$ Our patient was confirmed to have TB as the cause of granulomas based on positive multiplex PCR for Mycobacterium tuberculosis in kidney tissue. Glucocorticoids independently increase the risk for developing $\mathrm{TB}^{7}$ and also cause overwhelming disease in those affected with TB, if given without antituberculous therapy. Nevertheless, in conjunction with antituberculous therapy, glucocorticoids lead to beneficial effects in certain forms of TB. While common guidelines do not specifically recommend corticosteroids in treating tuberculous GIN, treatment usually comprises of antituberculous therapy with or without steroids. Whereas some advocate a combination of corticosteroids with antituberculous drugs, ${ }^{8}$ others do not. ${ }^{4}$ We have used corticosteroid with antituberculous drugs and gradually added MMF in our patient with good control of both TB infection and SLE activity.

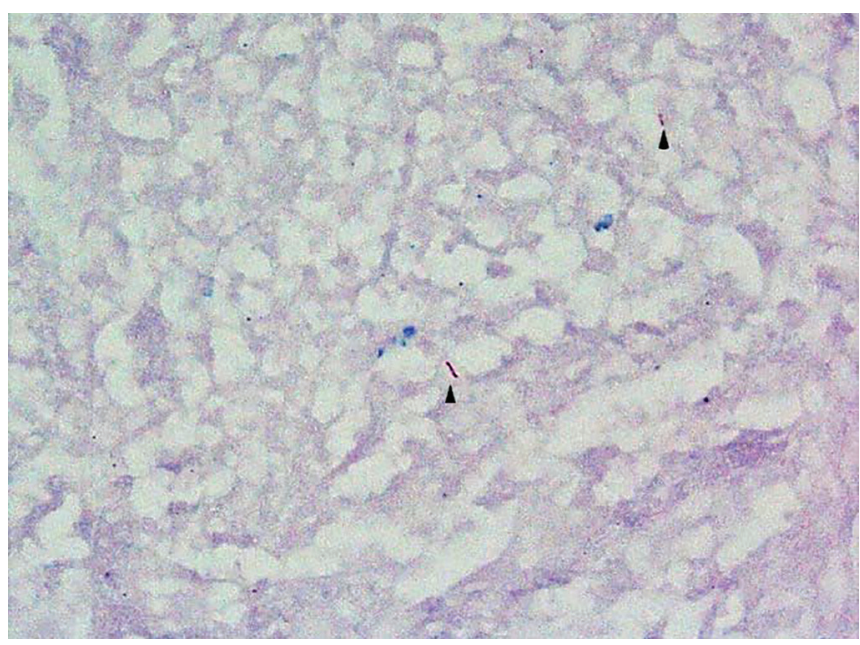

Fig. 5: Ziehl-Neelsen stain of the lung tissue $\times 100$-lung tissue showing red colored rod-shaped acid-fast bacilli (arrowheads)

\section{Conclusion}

A patient with an initial diagnosis of SLE can have also harbor TB at the same time. Molecular methods help in appropriate diagnosis of renal tuberculous granulomas. Whenever present, watchful observation, and treatment with steroids and ATT in the initial phase followed by an escalation of immunosuppression later might help treat both the disease entities.

\section{References}

1. Hou C-L, Tsai Y-C, Chen L-C, et al. Tuberculosis infection in patients with systemic lupus erythematosus: pulmonary and extra-pulmonary infection compared. Clin Rheumatol 2008;27(5):557-563. DOI: 10.1007/s10067-007-0741-8.

2. Ribeiro FM, Szyper-Kravitz M, Klumb EM, et al. Can lupus flares be associated with tuberculosis infection? Clin Rev Allergy Immunol 2010;38(2-3):163-168. DOI: 10.1007/s12016-009-8149-7.

3. Joss N, Morris S, Young B, et al. Granulomatous interstitial nephritis. Clin J Am Soc Nephrol CJASN 2007;2(2):222-230. DOI: 10.2215/ CJN.01790506.

4. Naidu GD, Ram R, Swarnalatha G, et al. Granulomatous interstitial nephritis: our experience of 14 patients. Indian J Nephrol 2013;23(6):415-418. DOI: 10.4103/0971-4065.120336.

5. Mignon F, Méry JP, Mougenot B, et al. Granulomatous interstitial nephritis. Adv Nephrol Necker Hosp 1984;13:219-245.

6. Agrawal V, Kaul A, Prasad N, et al. Etiological diagnosis of granulomatous tubulointerstitial nephritis in the tropics. Clin Kidney J 2015;8(5):524-530. DOI: 10.1093/ckj/sfv071.

7. Jick SS, Lieberman ES, Rahman MU, et al. Glucocorticoid use, other associated factors, and the risk of tuberculosis. Arthritis Rheum 2006;55(1):19-26. DOI: 10.1002/art.21705.

8. Chapagain A, Dobbie H, Sheaff M, et al. Presentation, diagnosis, and treatment outcome of tuberculous-mediated tubulointerstitia nephritis. Kidney Int 2011;79(6):671-677. DOI: 10.1038/ki.2010.482. 